\title{
The role of point of care ultrasound in prehospital critical care: a systematic review
}

Morten Thingemann Bøtker ${ }^{1,2^{*}}$ (D) Lars Jacobsen ${ }^{3,4}$, Søren Steemann Rudolph ${ }^{5,6}$ and Lars Knudsen ${ }^{2}$

\begin{abstract}
Background: In 2011, the role of Point of Care Ultrasound (POCUS) was defined as one of the top five research priorities in physician-provided prehospital critical care and future research topics were proposed; the feasibility of prehospital POCUS, changes in patient management induced by POCUS and education of providers. This systematic review aimed to assess these three topics by including studies examining all kinds of prehospital patients undergoing all kinds of prehospital POCUS examinations and studies examining any kind of POCUS education in prehospital critical care providers.

Methods and results: By a systematic literature search in MEDLINE, EMBASE, and Cochrane databases, we identified and screened titles and abstracts of 3264 studies published from 2012 to 2017. Of these, 65 studies were read in full-text for assessment of eligibility and 27 studies were ultimately included and assessed for quality by SIGN-50 checklists. No studies compared patient outcome with and without prehospital POCUS. Four studies of acceptable quality demonstrated feasibility and changes in patient management in trauma. Two studies of acceptable quality demonstrated feasibility and changes in patient management in breathing difficulties. Four studies of acceptable quality demonstrated feasibility, outcome prediction and changes in patient management in cardiac arrest, but also that POCUS may prolong pauses in compressions. Two studies of acceptable quality demonstrated that short (few hours) teaching sessions are sufficient for obtaining simple interpretation skills, but not image acquisition skills. Three studies of acceptable quality demonstrated that longer one- or two-day courses including hands-on training are sufficient for learning simple, but not advanced, image acquisition skills. Three studies of acceptable quality demonstrated that systematic educational programs including supervised examinations are sufficient for learning advanced image acquisition skills in healthy volunteers, but that more than 50 clinical examinations are required for expertise in a clinical setting.

Conclusion: Prehospital POCUS is feasible and changes patient management in trauma, breathing difficulties and cardiac arrest, but it is unknown if this improves outcome. Expertise in POCUS requires extensive training by a combination of theory, hands-on training and a substantial amount of clinical examinations - a large part of these needs to be supervised.
\end{abstract}

Keywords: Prehospital, Ultrasound, Critical care, Trauma, Cardiac arrest, Dyspnea, Point of care, Education, Systematic review

\footnotetext{
* Correspondence: botker@fastmail.fm

${ }^{1}$ Research and Development, Prehospital Emergency Medical Services,

Central Denmark Region, Aarhus, Denmark

${ }^{2}$ Department of Anesthesiology and Intensive Care Medicine, Aarhus

University Hospital, Aarhus, Denmark

Full list of author information is available at the end of the article
}

(c) The Author(s). 2018 Open Access This article is distributed under the terms of the Creative Commons Attribution 4.0 International License (http://creativecommons.org/licenses/by/4.0/), which permits unrestricted use, distribution, and reproduction in any medium, provided you give appropriate credit to the original author(s) and the source, provide a link to the Creative Commons license, and indicate if changes were made. The Creative Commons Public Domain Dedication waiver (http://creativecommons.org/publicdomain/zero/1.0/) applies to the data made available in this article, unless otherwise stated. 


\section{Background}

Prehospital Point-of-care Ultrasound (POCUS) can potentially improve patient outcome and the role of POCUS was defined as one the top five research priorities in physician-provided prehospital critical care in 2011 [1]. Three key research questions were identified; 1) which ultrasound examinations can be reliably transferred to the prehospital setting? 2) how does prehospital ultrasound affect patient management and the patient pathway? and 3) how should providers achieve and maintain specific ultrasound skills.

Although previous reviews have been positive towards the feasibility of prehospital POCUS, they were unable to demonstrate improved patient outcomes with POCUS $[2,3]$. This was mainly due to very limited and heterogeneous literature of low quality lacking patient centered outcome measures. Lack of evidence of improved patient outcomes, equipment costs and training difficulties are considered significant barriers to widespread use of prehospital ultrasound [4]. Prehospital patient categories with time-critical conditions as defined by the first hour quintet may benefit from improved early diagnostics (i.e. cardiac arrest, chest pain, stroke, respiratory failure, and severe trauma) [5]. Prehospital POCUS may also alter the patient pathway for other patient groups, which may be beneficial to both the patient and the health care system.

Thus, the aim of this study was to answer the three previously defined research questions by performing a systematic review on clinical use of prehospital POCUS and on prehospital POCUS education.

\section{Methods}

This was a commissioned systematic review on the role of POCUS in prehospital critical care conducted according to the Preferred Reporting Items for Systematic Reviews and Meta-Analyses (PRISMA) guidelines. No formal registration was performed.

\section{Eligibility criteria}

We included studies examining all types of patients of all ages undergoing a prehospital ultrasound examination and studies examining all types of ultrasound education in all types of prehospital critical care providers. Only interventional studies (randomized and non-randomized), observational controlled and un-controlled studies and studies of diagnostic accuracy were included. Only studies published in full-text in English were included.

\section{Outcome measures}

The primary outcome for clinical studies was patient survival within the study period. Secondary outcomes were changes in patient management, diagnostic accuracy, feasibility of the examinations and agreement between providers and experts. The primary outcome for educational studies was image acquisition skills. Secondary outcomes were image interpretation skills and theoretical knowledge.

\section{Information sources}

As commissioned by the journal, we included studies published from January 1st, 2012. We included studies indexed in MEDLINE, EMBASE, and Cochrane Central Register of Controlled Studies. In addition, we hand-searched all included studies for references and searched the ISI Web of Science: Science Citation Index for studies citing the included studies.

\section{Search strategy and study selection}

The search was conducted on April 24, 2017 according to the search strings supplied in the Additional file 1. Papers were imported into ENDNOTE X8 (Clarivate Analytics, Philadelphia, US) and duplicates were removed. Two reviewers (MTB and LK) independently screened papers by title and abstract and agreed on papers to assess for eligibility by their full-text version. The two reviewers then independently assessed which papers to include in the review based on their full-text. Discrepancies were solved by consensus. In case of doubt, an email was sent to the corresponding author for clarification.

\section{Data collection}

One reviewer (MTB) extracted the following study characteristics information into a standardized spreadsheet; author last name, publication date, study type, number of participants (providers and/or patients), aim of the study, and main results. For clinical studies, type of POCUS and provider-type (physicians, paramedics, nurses etc.) was extracted. For educational studies, the educational program used was extracted.

\section{Assessment of quality of evidence}

We used the relevant SIGN 50 checklists to assess the quality of the included studies and their risk of bias [6]. Two reviewers (SSR, LJ) independently assessed all points on the SIGN 50 checklist. When the reviewers agreed on a point, this assessment was considered final. Disagreements between reviewers were resolved by discussion using a third reviewer (MTB) as arbiter.

\section{Results}

We identified 3264 studies (Fig. 1). Of these, 27 studies were included in the review [7-33]. See the Additional file 1 for detailed reasons for exclusion following full-text assessment. Studies exclusively examining ultrasound in one of the first hour quintet patient groups are presented in Table 1, studies examining mixed 


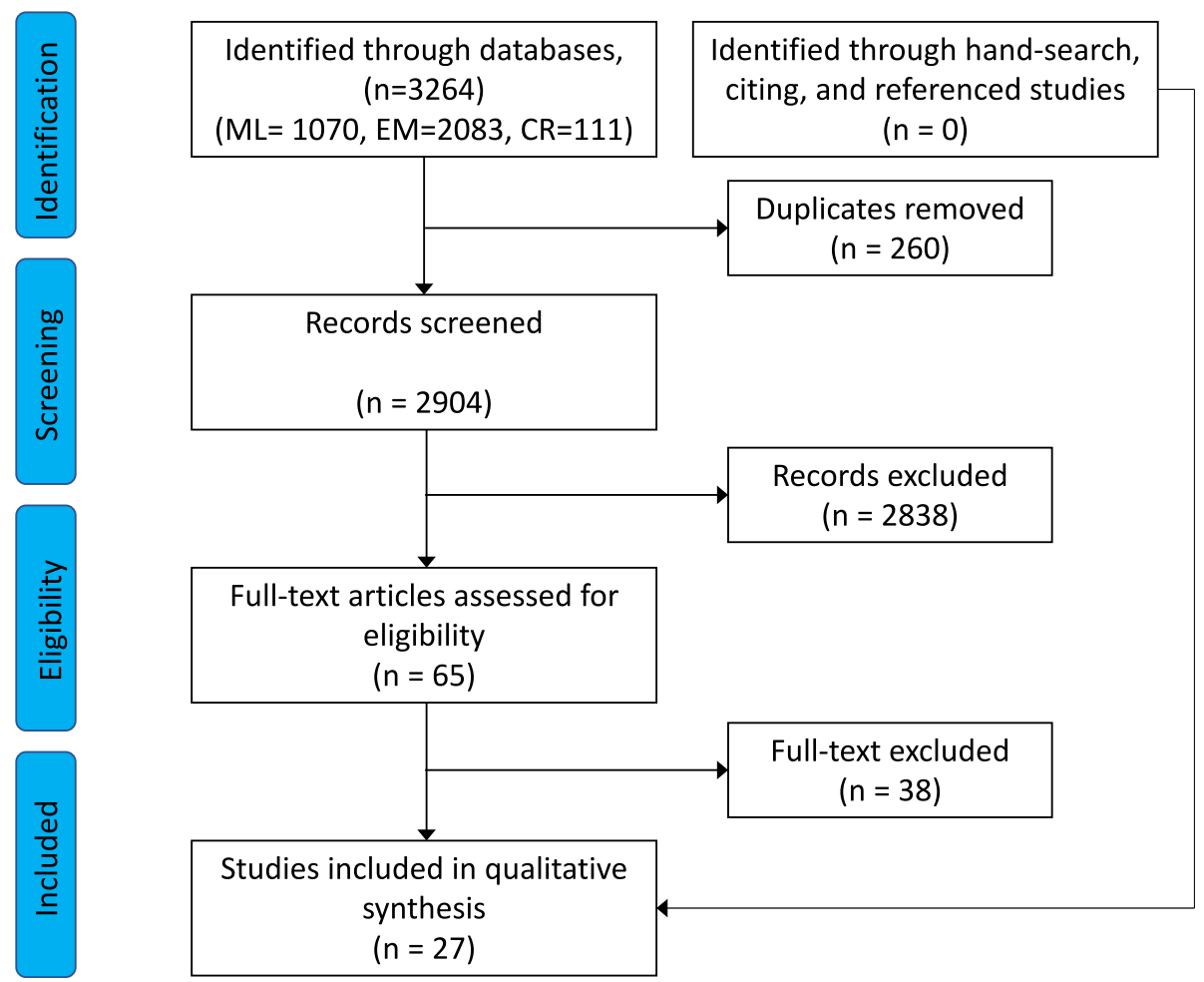

Fig. 1 PRISMA flow diagram

populations or POCUS for procedural guidance in Table 2, and studies examining the effect of education in Table 3. Details on the quality of evidence assessment can be found in the Additional file 1.

None of the included studies compared patient outcome or morbidity with and without application of POCUS.

\section{Cardiac arrest}

Three studies that were all of acceptable quality exclusively examined ultrasound in cardiac arrest patients and demonstrated feasibility of $80-100 \%$ [7, 27, 29]. One study demonstrated a high positive predictive value of cardiac standstill for death at $97.5 \%$ when assessed by physicians [7]. POCUS performed by paramedics during pulse-checks led to prolonged pauses in compressions in another study [27]. The last study demonstrated that paramedics were able to discriminate between cardiac activity and standstill [29]. Another study of acceptable quality examined physician-based POCUS in both trauma and cardiac arrest patients and demonstrated frequent changes in patient management, among others a decision to cease resuscitation in 9 of 31 (29\%) of cardiac arrest patients [18].

\section{Chest pain}

None of the included studies specifically examined patients with chest pain.

\section{Stroke}

One study examined transcranial ultrasound conducted by expert neurologists and demonstrated a high specificity for major stroke, but was rejected (see details of the quality of evidence assessment in the Additional file 1) [17].

\section{Breathing difficulties}

Three studies evaluated POCUS conducted by physicians in patients with breathing difficulties $[20,21$, $30]$. One study of acceptable quality demonstrated $100 \%$ feasibility for simplified lung ultrasound evaluation of B-lines and a high negative predictive value of $94 \%$, but a lower positive predictive value of $77 \%$ for congestive heart failure [20]. One study of acceptable quality demonstrated that pleural effusion is a $100 \%$ sensitive marker for congestive heart failure and that POCUS in dyspneic patients causes additional therapeutic consequences in $25 \%$ of patients [21]. The last study examining the use of B-lines by lung ultrasound to monitor the effect of treatment in heart failure patients was rejected (see details of the quality of evidence assessment in the Additional file 1) [30].

\section{Trauma}

Three studies exclusively examined trauma patients [12, $24,32]$. One study of acceptable quality examined each 


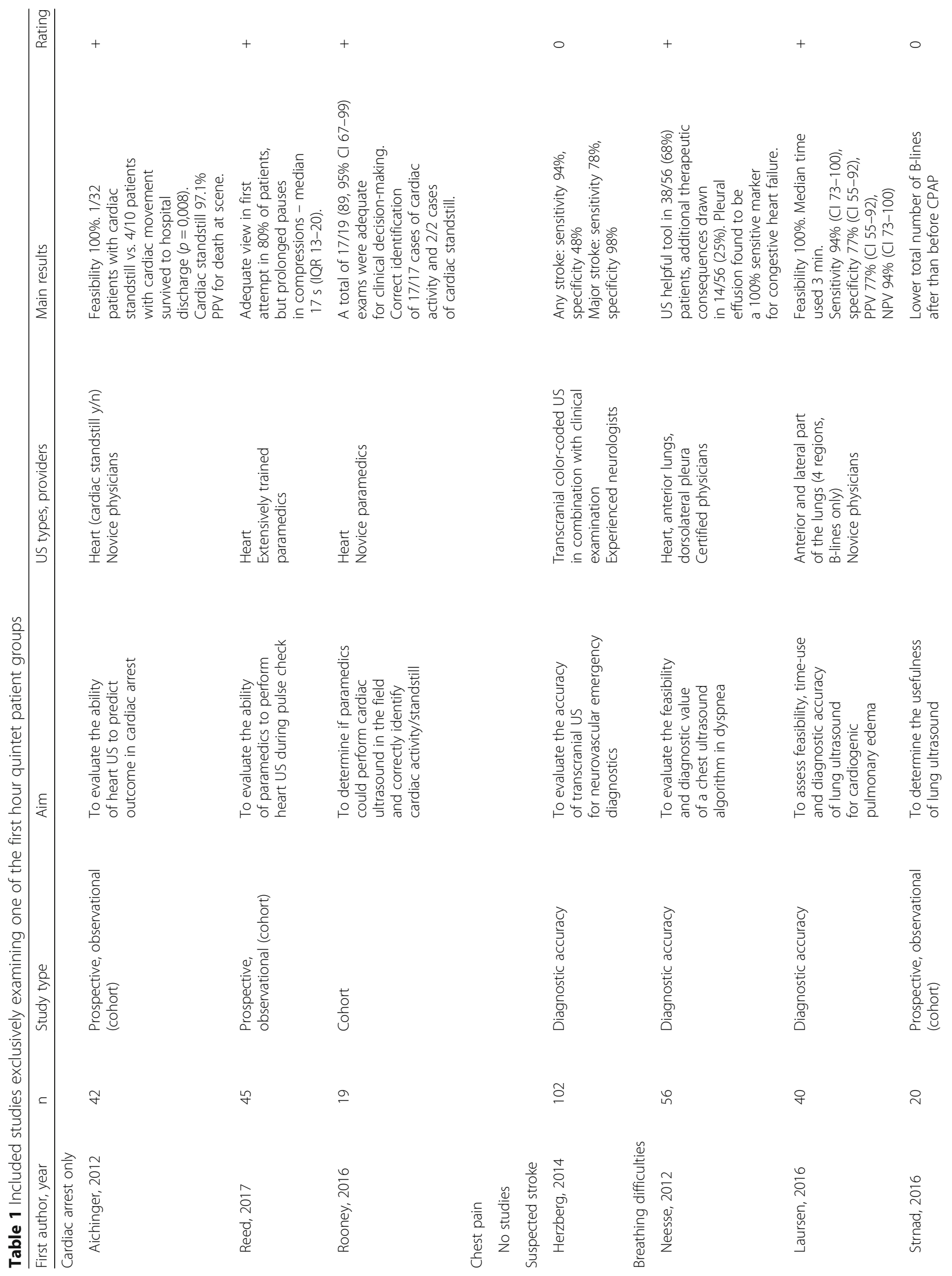


Bøtker et al. Scandinavian Journal of Trauma, Resuscitation and Emergency Medicine (2018) 26:51

Page 5 of 14

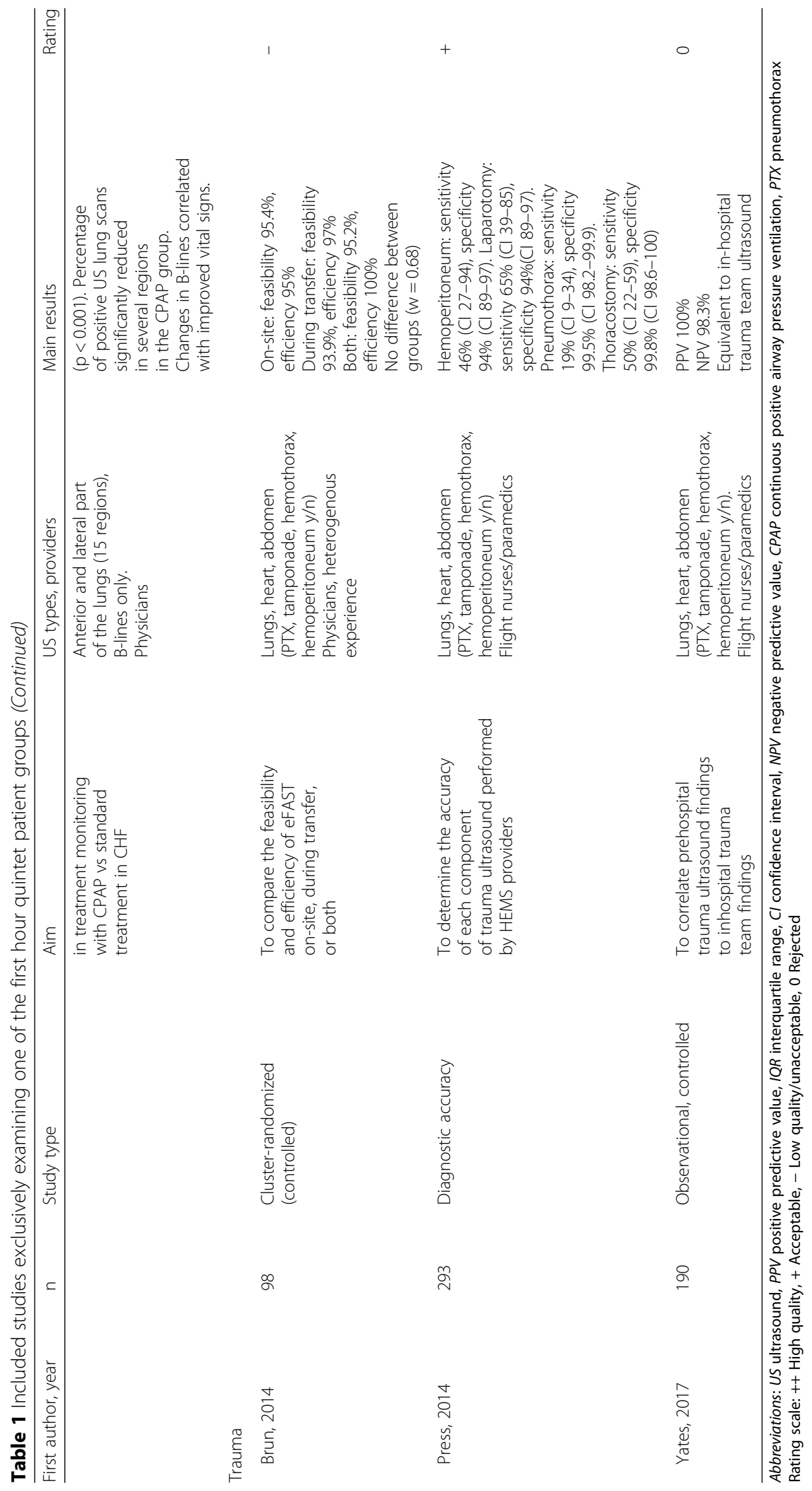


Bøtker et al. Scandinavian Journal of Trauma, Resuscitation and Emergency Medicine (2018) 26:51

Page 6 of 14

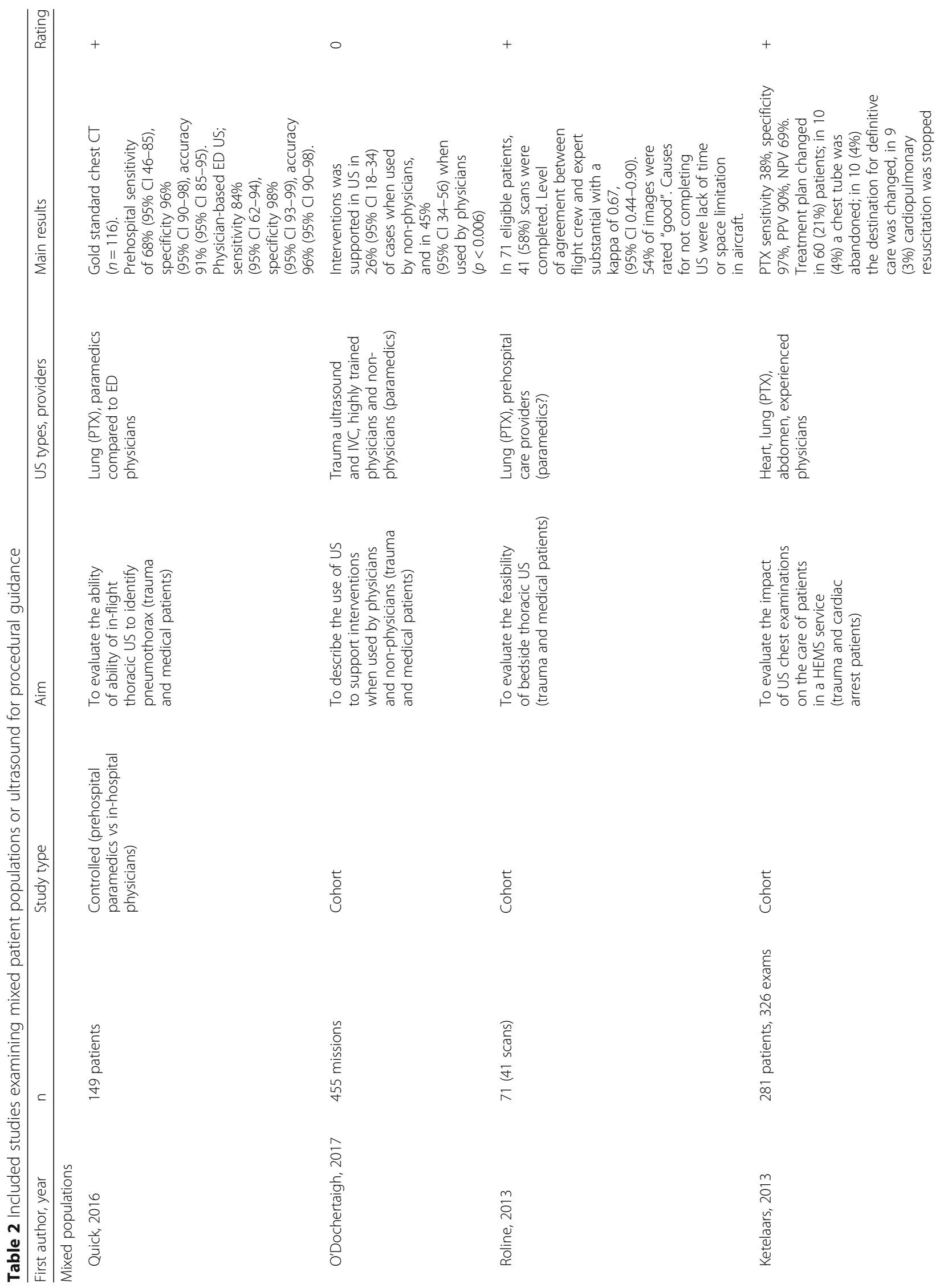




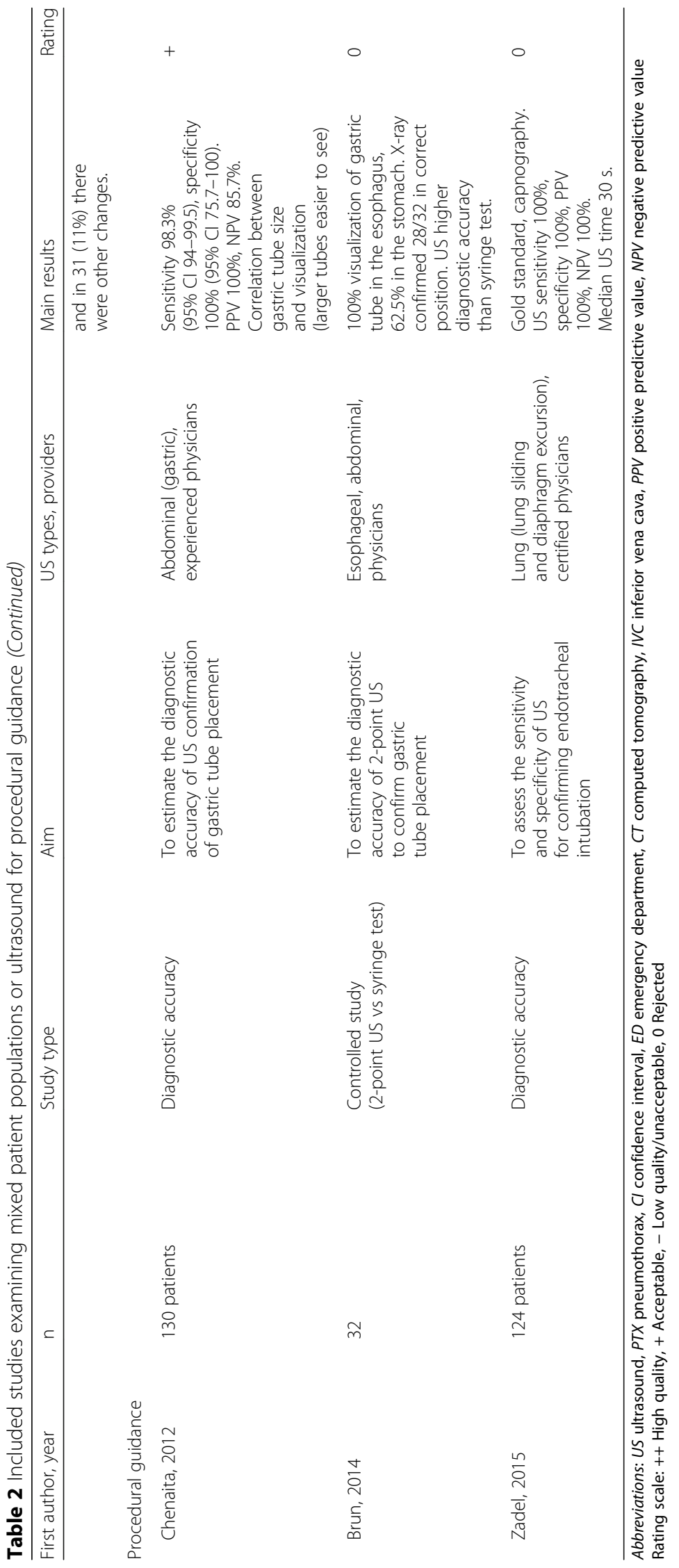


component of the trauma ultrasound examination and demonstrated a positive predictive value of $90 \%$ and a negative predictive value of $98 \%$ for a required intervention due to pneumothorax, a positive predictive value of $50 \%$ with a negative predictive value of $96 \%$ for a need for laparotomy due to intraabdominal free fluid, but had an insufficient amount of pericardial effusions for reliability on this part [24]. The last two studies exclusively in trauma patients were either rejected or assessed to be of low quality (see details of the quality of evidence assessment in the Additional file 1) [12, 32]. Three studies of acceptable quality examined both trauma and medical patients and demonstrated a high level of agreement between prehospital examinations and in-hospital ultrasound assessment by expert sonographers and a change in treatment in $20 \%$ of trauma patients $[18,26,28]$. A study comparing intervention support in both trauma and medical patients when ultrasound was used by physicians and non-physicians was rejected (see details of the quality of evidence assessment in the Additional file 1) [22].

\section{Education}

Eleven studies examined POCUS education in prehospital critical care providers $[8-11,14,16,19,23,25,29$, 31]. Three of these were either rejected or assessed to be of low quality (see details of the quality of evidence assessment in the Additional file 1) [10, 16, 31].

Two studies examining short courses were of acceptable quality $[8,29]$. One demonstrated that a simple one-hour lecture improves theoretical knowledge among paramedics [8]. The other demonstrated that $2 \mathrm{~h}$ theory and $1 \mathrm{~h}$ hands-on training in paramedics with no prior ultrasound experience lead to images useful for clinical interpretation in $89 \%$ of cardiac arrest patients and correct identification of cardiac activity and cardiac standstill [29].

Three studies examining 1- or 2 day courses were of acceptable quality $[14,19,23]$. One demonstrated that theoretical knowledge, image interpretation skills and a structured observation of ultrasound examination skills in lung, heart, and abdominal ultrasound, could be improved by $2 \mathrm{~h}$ e-learning and $4 \mathrm{~h}$ hands-on course [19]. One demonstrated that after completing a two-day course, cardiac image acquisition skills were only moderate and agreement with experts was weak for left ventricular function, right ventricular size, and pericardial effusion and very weak for inferior vena cava assessment [14]. The last demonstrated that there was no difference in neither image acquisition skills nor theoretical knowledge scores when comparing traditional trauma ultrasound training to simulator-based training or both [23].

Three studies of acceptable quality examined the effect of longer educational programs $[9,11,25]$. One study examined a program comprising 1-day course with $2 \mathrm{~h}$ lectures and $4 \mathrm{~h}$ hands-on followed by at least four supervised examinations in real-life patients, $60-120 \mathrm{~min}$ e-learning and a number of unsupervised real-life examinations and demonstrated that 27 and 28 of 33 paramedics were able to pass a structured clinical exam and a theoretical exam, respectively [25]. Another study examined the effect of a program comprising $4 \mathrm{~h}$ e-learning, 1-day hands-on course, 10 supervised examinations in real-life patients and a number of unsupervised examinations and demonstrated $98 \%$ image acquisition ability after the program and that $21 / 21(100 \%)$ physicians used ultrasound in the prehospital setting after the program [11]. The last study compared image acquisition skills among experienced and inexperienced physician providers (defined as more or less than 50 examinations after initial training) and demonstrated a highly significant difference for all evaluated items [9].

\section{Procedural guidance}

Two studies evaluated the use of ultrasound to confirm gastric tube placement $[13,15]$. One was rejected [13]. The other demonstrated high sensitivity and specificity of gastric ultrasound [15]. One study evaluating the effect of lung ultrasound to confirm endotracheal intubation was rejected (see details of the quality of evidence assessment in the Additional file 1) [33].

\section{Discussion}

The main finding of this review is that considerable amounts of literature on both clinical use of prehospital POCUS and POCUS education for prehospital providers has been published since 2011, indicating a growing interest in prehospital POCUS. The most recent literature does not provide evidence of outcome improvement, but supports the use of POCUS in trauma and breathing difficulties, calls for caution in cardiac arrest, and indicates that extensive training efforts are needed for providers to obtain the necessary skills.

Previous reviews on prehospital ultrasound have pointed to a high risk of bias in the published studies and to the lack of evidence for outcome improvements $[2,3]$. The authors of this review still share this concern, but consider the quality of studies included in this review as improved. Nevertheless, studies are still very heterogeneous and of variable scientific quality and the literature lacks patient centered outcome measures.

\section{Which ultrasound examinations can be reliably transferred to the prehospital setting?}

Prehospital POCUS of the lungs for the diagnosis of pneumothorax has a moderate diagnostic accuracy and shows good agreement with experts [18, 24, 26, 28]. Positive predictive values ranges from 80 to $90 \%$ and 


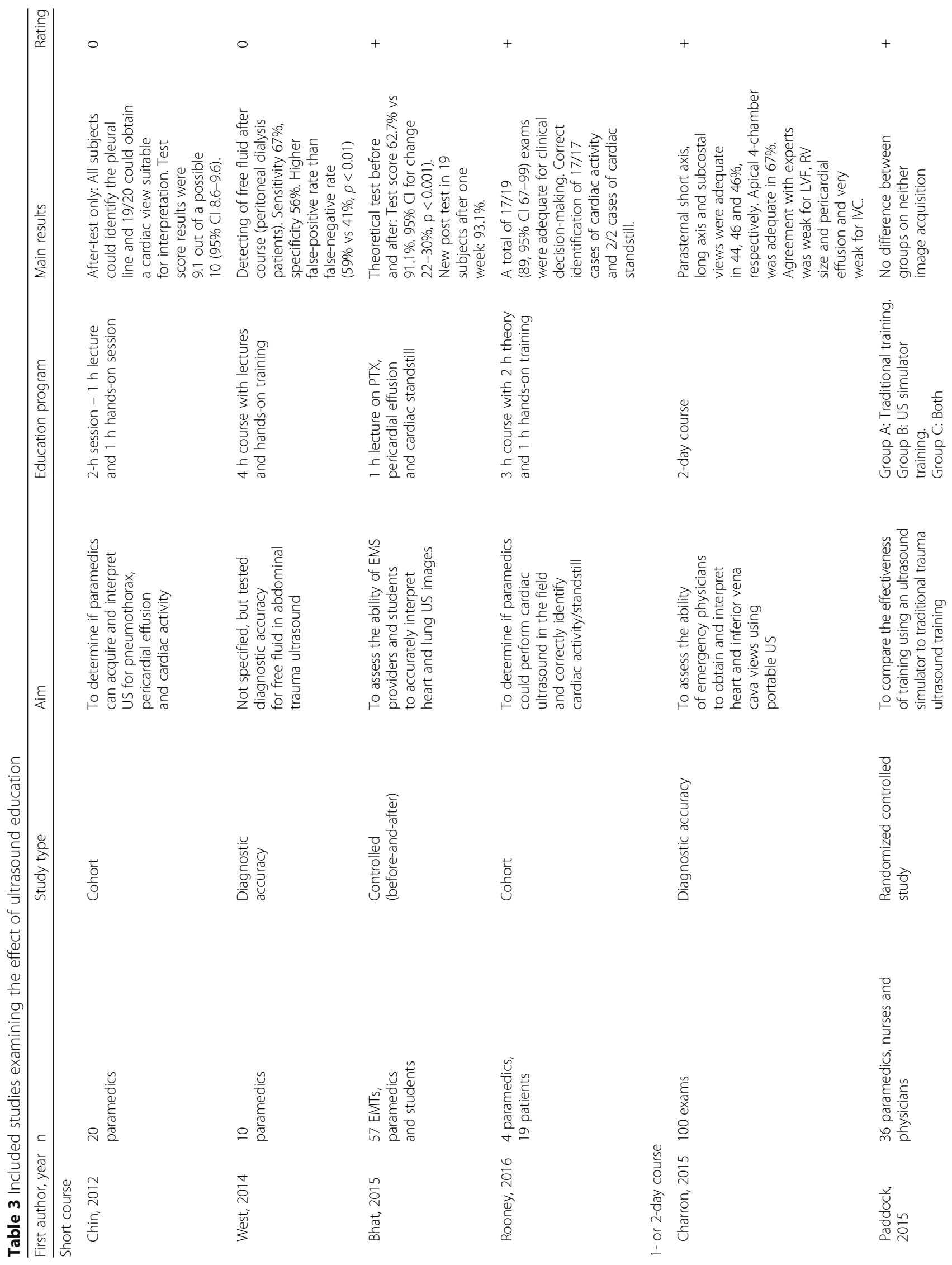


Bøtker et al. Scandinavian Journal of Trauma, Resuscitation and Emergency Medicine (2018) 26:51

Page 10 of 14

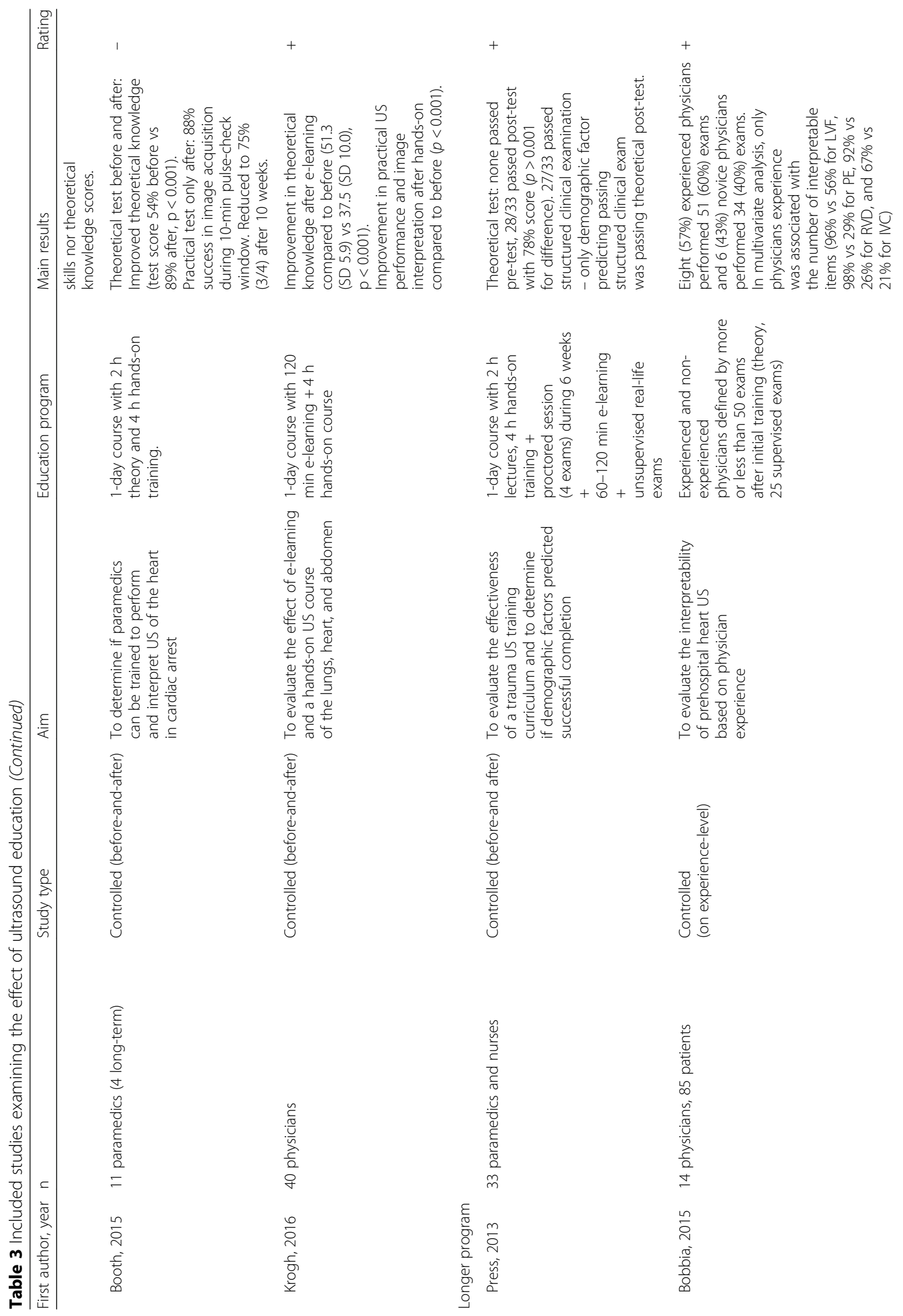




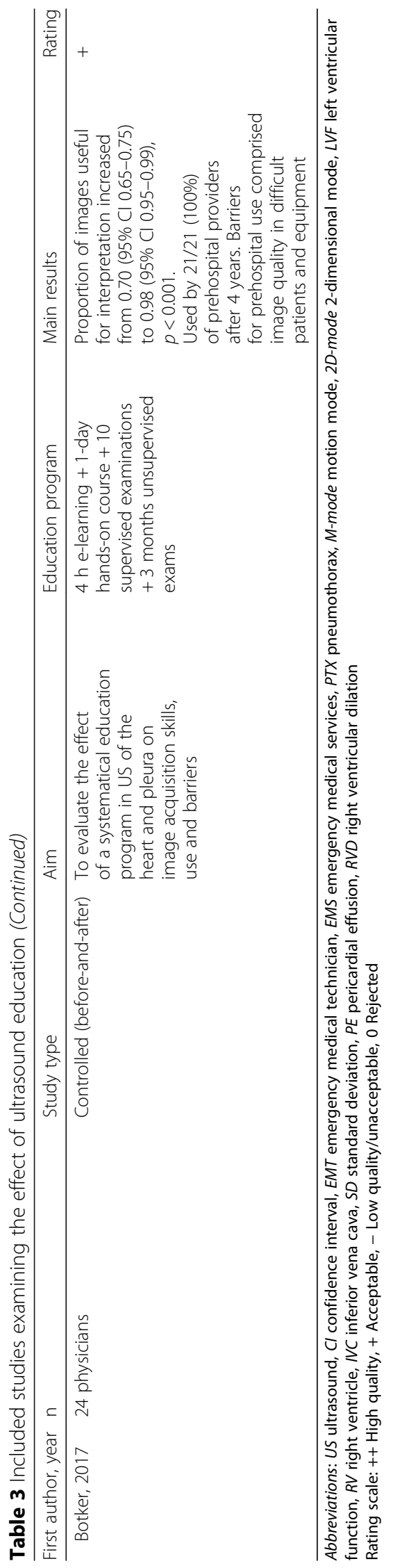


negative predictive values from 69 to $90 \%$. The same patterns apply to prehospital trauma ultrasound, although positive predictive value is generally lower for hemoperitoneum (around 50\%) than for pneumothorax [24]. A positive POCUS finding is highly predictive of a need for intervention and seems useful for prehospital triage $[18,24]$. The negative predictive values are not sufficiently high to recommend POCUS-based rule-out of serious injuries.

Prehospital POCUS of the lungs to diagnose congestive heart failure in patients with breathing difficulties displays high negative predictive value but lower positive predictive value and is reliable for rule-out, but not rule-in of congestive heart failure [20]. The addition of POCUS of the pleura may improve the positive predictive value for the diagnosis of congestive heart failure [21]. Recent studies conducted in in-hospital settings suggest that supplementing POCUS of the lungs with POCUS of the heart may further improve the positive predictive value and reduce the time to correct diagnosis $[34,35]$.

Prehospital POCUS of the heart is feasible and reliable for assessing simple dichotomous questions in cardiac arrest like "cardiac activity yes/no", but may cause prolonged pauses in compressions during cardiopulmonary resuscitation $[7,27,29]$. The ability to assess more complex measures like pericardial effusion, left ventricular function, and right ventricular dilation requires extensive training and clinical ultrasound experience $[9,14]$. There were no studies examining prehospital ultrasound in chest pain patients during the study period, but a recently published study demonstrated that ultrasound may also be used for early diagnosis of non-ST-elevation myocardial infarction in patients suspected of acute coronary syndrome [36].

\section{How does prehospital ultrasound affect patient management and the patient pathway?}

Prehospital POCUS predicts the need for interventions and causes changes in patient management in both trauma, cardiac arrest, and breathing difficulties [18, 21, 24]. But, it is unknown if these changes improve patient outcomes. Since the inclusion period of this review, a secondary analysis of an included study was published $[37,22]$. This study demonstrated that interventions were more likely to be supported with ultrasound in patients with markers of high acuity than in patients with presumed low-grade disease [37]. We do however question the practice of ceasing resuscitation based on cardiac standstill used in one study [18]. Early studies on this were promising $[38,39]$. Yet, there are survivors following cardiac standstill in both recent and previous studies, indicating that this decision should not be based on POCUS alone [7, 40, 39].

\section{How should providers achieve and maintain specific ultrasound skills?}

Lectures seem efficient for obtaining the simplest of image interpretation skills, while image acquisition skills require hands-on training $[8,19,29]$. The type of training used (i.e. traditional or simulation training) seems less important [23]. Systematic educational programs comprising some sort of theory (e-learning and/or lectures), hands-on training, supervised examinations, and unsupervised clinical use makes it possible to consistently produce images useful for interpretation in healthy volunteers $[11,25]$. Physician experience seems to affect especially the interpretability of POCUS images of the heart after initial hands-on training and 50 examinations greatly improves image acquisition skills in real-life patients [9]. This is in accordance with a recent in-hospital study demonstrating that for most examination types, between 50 and 75 results in both excellent interpretation and good image quality in actual patients [41].

\section{Future research questions}

Future research should address the gap in the literature demonstrating a beneficial effect of POCUS on patient centered outcome measures (improved triage, improved treatment, length-of-stay, and when possible mortality). But, to translate diagnostic accuracy into clinical utility we need to take one step back from the protocols. POCUS protocols have been defined a priori, and there is a tendency in the literature to promote specific ultrasound protocols. This is research in reverse order. When dealing with a specific patient with a specific medical history, symptoms and objective findings, some clinical questions (or differential diagnoses) arise - some of these may be answered by ultrasound. Thus, more studies on the diagnostic accuracy on specific components of a POCUS examination (such as B-lines, pleural effusion, impaired LV function) in patients with specific symptomatology (like chest pain, dyspnea, cardiac arrest, etc.) are needed to clarify which findings are key and which examinations are a waste of valuable time [42]. Only then can good controlled trials examining decision-making with and without ultrasound be planned. The Press et al. study examining sensitivity and specificity for each of the components in the trauma ultrasound examination in relation to both the relevant pathology and the associated intervention is a good example of the types of studies needed for other patient categories [24].

There is an ethical dilemma in educating prehospital critical care providers in ultrasound and randomize patients to either have the examination or not. This may be overcome by examining outcome in specific patient groups (such as abdominal aortic aneurism) in case-control studies where patients triaged directly to a specialized center by prehospital ultrasound is compared 
to patients admitted to local hospital and secondarily transferred, although this type of studies carries inherent risks of bias. Another way of overcoming this could be to perform cluster-randomized studies in emergency medical services where ultrasound is not already implemented. In addition, the distance to nearest hospital (and/or specialized center) may affect the value of prehospital ultrasound. Which examinations can effectively change patient management depends highly upon the local setting and organization of both prehospital and hospital care. Thus, distance and time in the emergency medical services are relevant issues for future POCUS research.

There is still a paucity of literature aiming at determining the number of examinations needed for clinical proficiency. This may be addressed by linking individual level experience to the quality of images and the correctness of clinical interpretations when compared to expert assessment.

\section{Limitations}

Publication bias may have led to studies with neutral findings not being included - this may have been exaggerated by the choice to only include studies published in English. Especially the educational section may suffer from publication bias and conclusions must be interpreted with caution. Although the use of checklists for study quality assessment is generally recommended, the studies included in this review were very heterogeneous and we had difficulties deciding which checklists to use. Many educational studies were "before-and-after" studies. The results of this kind of study generally must be interpreted with caution due to a high risk of confounding and bias in favor of the intervention.

\section{Conclusion}

Prehospital POCUS remains unexamined in a wide range of patient groups. Prehospital POCUS seems feasible and changes patient management in trauma and breathing difficulties. POCUS is also feasible in cardiac arrest but may cause prolonged pauses in compressions. It is unknown how prehospital POCUS affects patient outcome. The best available evidence suggests that specific POCUS skills can be achieved by a combination of theoretical education, hands-on teaching, and more than 50 clinical examinations of which a large part are supervised.

\section{Additional file}

Additional file 1: Search strings Table S1. Studies excluded based on full text Table S2. SIGN 50 checklist of cohort studies included in the review Table S3. SIGN 50 checklist of controlled studies included in the review 5 Table S4. SIGN 50 checklist of diagnostic accuracy studies included in the review references. (PDF $229 \mathrm{~kb}$ )

\section{Abbreviations}

POCUS: Point of Care Ultrasound; PRISMA: Preferred Reporting Items for Systematic Reviews and Meta-Analyses

\section{Acknowledgements}

Not applicable.

\section{Funding}

This study was initiated by the authors on request from Scandinavian Journal of Trauma, Resuscitation and Emergency Medicine and did not receive any external funding.

\section{Availability of data and materials}

Not applicable.

\section{Authors' contributions}

MTB, LK, SSR, and LJ made the study protocol. MTB and LK conducted the literature search. SSR and $L$ performed the assessment of study quality with MTB as third reviewer. MTB drafted the manuscript and SSR, LJ and LK revised the manuscript and approved it before submission.

Ethics approval and consent to participate

Not applicable.

Consent for publication

Not applicable.

\section{Competing interests}

MTB receives royalties for e-learning produced for USabcd.org. LK is co-owner of USabcd.org. SR and $\sqcup$ reported no competing interests.

\section{Publisher's Note}

Springer Nature remains neutral with regard to jurisdictional claims in published maps and institutional affiliations.

\section{Author details}

${ }^{1}$ Research and Development, Prehospital Emergency Medical Services, Central Denmark Region, Aarhus, Denmark. ${ }^{2}$ Department of Anesthesiology and Intensive Care Medicine, Aarhus University Hospital, Aarhus, Denmark. ${ }^{3}$ Department of Research and Development, Norwegian Air Ambulance Foundation, Drøbak, Norway. ${ }^{4}$ Air Ambulance department, Sorlandet Hospital Thrust, Arendal, Norway. ${ }^{5}$ Centre of Head and Orthopedics, Department of Anaesthesia, Rigshospitalet, Denmark. ${ }^{6}$ The Emergency Medical Services, Copenhagen, Denmark.

Received: 11 September 2017 Accepted: 11 June 2018

Published online: 26 June 2018

\section{References}

1. Fevang E, Lockey D, Thompson J, Lossius HM. The top five research priorities in physician-provided pre-hospital critical care: a consensus report from a European research collaboration. Scand J Trauma Resusc Emerg Med. 2011;19:57.

2. Brooke M, Walton J, Scutt D. Paramedic application of ultrasound in the management of patients in the prehospital setting: a review of the literature. Emerg Med J. 2010;27(9):702-7.

3. Rudolph SS, Sorensen MK, Svane C, Hesselfeldt R, Steinmetz J. Effect of prehospital ultrasound on clinical outcomes of non-trauma patients-a systematic review. Resuscitation. 2014;85(1):21-30.

4. Taylor J, McLaughlin K, McRae A, Lang E, Anton A. Use of prehospital ultrasound in North America: a survey of emergency medical services medical directors. BMC Emerg Med. 2014;14:6.

5. Fischer M, Kamp J, Garcia-Castrillo Riesgo L, Robertson-Steel I, Overton J, Ziemann A, et al. Comparing emergency medical service systems-a project of the European Emergency Data (EED) Project. Resuscitation. 2011;82(3):285-93.

6. Harbour R, Miller J. A new system for grading recommendations in evidence based guidelines. BMJ. 2001;323(7308):334-6.

7. Aichinger G, Zechner PM, Prause G, Sacherer F, Wildner G, Anderson $\mathrm{CL}$, et al. Cardiac movement identified on prehospital echocardiography 
predicts outcome in cardiac arrest patients. Prehosp Emerg Care. 2012; 16(2):251-5.

8. Bhat SR, Johnson DA, Pierog JE, Zaia BE, Williams SR, Gharahbaghian L. Prehospital evaluation of effusion, pneumothorax, and standstill (PEEPS): Point-of-care ultrasound in emergency medical services. West J Emerg Med. 2015;16(4):503-9.

9. Bobbia X, Pradeilles C, Claret PG, Soullier C, Wagner P, Bodin Y, et al. Does physician experience influence the interpretability of focused echocardiography images performed by a pocket device? Scand I Trauma Resusc Emerg Med. 2015:23:52.

10. Booth KL, Reed MJ, Brady S, Cranfield KR, Kishen R, Letham K, et al. Training paramedics in focussed echo in life support. Eur J Emerg Med. 2015;22(6):430-5.

11. Botker MT, Vang ML, Grofte T, Kirkegaard H, Frederiksen CA, Sloth E. Implementing point-of-care ultrasonography of the heart and lungs in an anesthesia department. Acta Anaesthesiol Scand. 2017;61(2):156-65.

12. Brun PM, Bessereau J, Chenaitia H, Pradel AL, Deniel C, Garbaye G, et al. Stay and play eFAST or scoop and run eFAST? That is the question! Am J Emerg Med. 2014;32(2):166-70.

13. Brun PM, Chenaitia H, Lablanche C, Pradel AL, Deniel C, Bessereau J, et al. 2point ultrasonography to confirm correct position of the gastric tube in prehospital setting. Mil Med. 2014;179(9):959-63.

14. Charron C, Templier F, Goddet NS, Baer M, Vieillard-Baron A. Difficulties encountered by physicians in interpreting focused echocardiography using a pocket ultrasound machine in prehospital emergencies. Eur J Emerg Med. 2015;22(1):17-22.

15. Chenaitia H, Brun PM, Querellou E, Leyral J, Bessereau J, Aime C, et al. Ultrasound to confirm gastric tube placement in prehospital management. Resuscitation. 2012;83(4):447-51.

16. Chin EJ, Chan CH, Mortazavi R, Anderson CL, Kahn CA, Summers S, et al. A pilot study examining the viability of a prehospital assessment with ultrasound for emergencies (PAUSE) protocol. J Emerg Med. 2013;44(1):142-9.

17. Herzberg M, Boy S, Holscher T, Ertl M, Zimmermann M, Ittner KP, et al. Prehospital stroke diagnostics based on neurological examination and transcranial ultrasound. Crit Ultrasound J. 2014;6(1):3.

18. Ketelaars R, Hoogerwerf N, Scheffer GJ. Prehospital chest ultrasound by a dutch helicopter emergency medical service. J Emerg Med. 2013;44(4):811-7.

19. Krogh CL, Steinmetz J, Rudolph SS, Hesselfeldt R, Lippert FK, Berlac PA, et al. Effect of ultrasound training of physicians working in the prehospital setting. Scand J Trauma Resusc Emerg Med. 2016;24:99.

20. Laursen CB, Hanselmann A, Posth S, Mikkelsen S, Videbaek L, Berg H. Prehospital lung ultrasound for the diagnosis of cardiogenic pulmonary oedema: a pilot study. Scand J Trauma Resusc Emerg Med. 2016;24:96.

21. Neesse A, Jerrentrup A, Hoffmann S, Sattler A, Gorg C, Kill C, et al. Prehospital chest emergency sonography trial in Germany: a prospective study. Eur J Emerg Med. 2012;19(3):161-6.

22. O'Dochartaigh D, Douma M, MacKenzie M. Five-year Retrospective Review of Physician and Non-physician Performed Ultrasound in a Canadian Critical Care Helicopter Emergency Medical Service. Prehosp Emerg Care. 2017;21(1):24-31.

23. Paddock MT, Bailitz J, Horowitz R, Khishfe B, Cosby K, Sergel MJ. Disaster response team FAST skills training with a portable ultrasound simulator compared to traditional training: Pilot study. West J Emerg Med. 2015;16(2):325-30.

24. Press GM, Miller SK, Hassan IA, Alade KH, Camp E, Del Junco D, et al. Prospective evaluation of prehospital trauma ultrasound during aeromedical transport. J Emerg Med. 2014;47(6):638-45.

25. Press GM, Miller SK, Hassan IA, Blankenship R, del Junco D, Camp E, et al. Evaluation of a training curriculum for prehospital trauma ultrasound. J Emerg Med. 2013;45(6):856-64.

26. Quick JA, Uhlich RM, Ahmad S, Barnes SL, Coughenour JP. In-flight ultrasound identification of pneumothorax. Emerg Radiol. 2016;23(1):3-7.

27. Reed MJ, Gibson L, Dewar A, Clegg GR, Black P, Clegg GR. Introduction of paramedic led Echo in Life Support into the pre-hospital environment: The PUCA study. Resuscitation. 2017;112:65-9.

28. Roline CE, Heegaard WG, Moore JC, Joing SA, Hildebrandt DA, Biros MH, et al. Feasibility of bedside thoracic ultrasound in the helicopter emergency. medical services setting. Air Med J. 2013;32(3):153-7.

29. Rooney KP, Lahham S, Lahham S, Anderson CL, Bledsoe B, Sloane B, et al. Pre-hospital assessment with ultrasound in emergencies: implementation in the field. World J Emerg Med. 2016;7(2):117-23.
30. Strnad M, Prosen G, Borovnik Lesjak V. Bedside lung ultrasound for monitoring the effectiveness of prehospital treatment with continuous positive airway pressure in acute decompensated heart failure. Eur J Emerg Med. 2016;23(1):50-5.

31. West $B$, Cusser A, Etengoff $S$, Landsgaard $H$, LaBond V. The use of FAST scan by paramedics in mass-casualty incidents: a simulation study. Prehosp Disaster Med. 2014;29(6):576-9.

32. Yates JG, Baylous D. Aeromedical Ultrasound: The Evaluation of Point-ofcare Ultrasound During Helicopter Transport. Air Med J. 2017;

33. Zadel S, Strnad M, Prosen G, Mekis D. Point of care ultrasound for orotracheal tube placement assessment in out-of hospital setting Resuscitation. 2015;87:1-6.

34. Laursen CB, Sloth E, Lassen AT, Christensen R, Lambrechtsen J, Madsen PH, et al. Point-of-care ultrasonography in patients admitted with respiratory symptoms: a single-blind, randomised controlled trial. Lancet Respir Med. 2014;2(8):638-46.

35. Zanobetti M, Scorpiniti M, Gigli C, Nazerian P, Vanni S, Innocenti F, et al. Point-of-Care Ultrasonography for Evaluation of Acute Dyspnea in the ED. Chest. 2017;151(6):1295-301.

36. Bergmann I, Buttner B, Teut E, Jacobshagen C, Hinz J, Quintel M, et al. Prehospital transthoracic echocardiography for early identification of non-STelevation myocardial infarction in patients with acute coronary syndrome. Crit Care. 2018;22(1):29.

37. O'Dochartaigh D, Douma M, Alexiu C, Ryan S, Mackenzie M. Utilization Criteria for Prehospital Ultrasound in a Canadian Critical Care Helicopter Emergency Medical Service: Determining Who Might Benefit. Prehosp Disaster Med 2017;32(5):536-540.

38. Blaivas M, Fox JC. Outcome in cardiac arrest patients found to have cardiac standstill on the bedside emergency department echocardiogram. Acad Emerg Med. 2001;8(6):616-21.

39. Salen P, Melniker L, Chooljian C, Rose JS, Alteveer J, Reed J, et al. Does the presence or absence of sonographically identified cardiac activity predict resuscitation outcomes of cardiac arrest patients? Am J Emerg Med. 2005; 23(4):459-62.

40. Breitkreutz R, Price S, Steiger HV, Seeger FH, Ilper H, Ackermann $H$, et al. Focused echocardiographic evaluation in life support and peri-resuscitation of emergency patients: a prospective trial. Resuscitation. 2010;81 (11):1527-33.

41. Blehar DJ, Barton B, Gaspari RJ. Learning curves in emergency ultrasound education. Acad Emerg Med. 2015;22(5):574-82.

42. Bossuyt PM, Reitsma JB, Linnet K, Moons KG. Beyond diagnostic accuracy: the clinical utility of diagnostic tests. Clin Chem. 2012;58(12):1636-43.

\section{Ready to submit your research? Choose BMC and benefit from:}

- fast, convenient online submission

- thorough peer review by experienced researchers in your field

- rapid publication on acceptance

- support for research data, including large and complex data types

- gold Open Access which fosters wider collaboration and increased citations

- maximum visibility for your research: over $100 \mathrm{M}$ website views per year

At BMC, research is always in progress.

Learn more biomedcentral.com/submissions 